\title{
A Centralised Wi-Fi Management Framework for D2D Communications in Dense Wi-Fi Networks
}

\author{
Mirghiasaldin Seyedebrahimi, Alessandro Raschellà, \\ Faycal Bouhafs, Michael Mackay, Qi Shi \\ Department of Computer Science, Liverpool John Moores \\ University, Liverpool, U.K. \\ \{M.Seyedebrahimi, A.Raschella, F.Bouhafs, M.I.Mackay, \\ Q.Shi\}@ljmu.ac.uk
}

\author{
Mahmoud Hashem Eiza \\ School of Physical Sciences and Computing \\ University of Central Lancashire \\ Preston, U.K. \\ MHashemEiza@uclan.ac.uk
}

\begin{abstract}
In Wi-Fi networks, Device-to-Device (D2D) communications aim to improve the efficiency of the network by supporting direct communication between users in close proximity. However, in a congested Wi-Fi network, establishing D2D connections through a locally managed self-organising approach will intensify the congestion and reduce the scalability of the solution. Therefore, a centralised management approach must be involved in orchestrating those actions to guarantee the sufficiency of D2D communications. In this paper, we propose a novel management framework for D2D communications in dense Wi-Fi networks. The proposed framework employs a SoftwareDefined Networking (SDN) based centralised controller in synergy with a novel Access Point (AP) channel assignment process. This framework is designed to proactively establish and manage D2D connections in Wi-Fi networks considering the available radio resources and the effect of the subsequent interference. Thus, improving the overall performance of the network and providing users with higher data rate. Through simulation, we validate the effectiveness of the proposed framework and demonstrate how D2D deployment considerably improves the Wi-Fi network efficiency especially when the data rate requirements are high. Furthermore, we show that our proposed framework achieves better performance than the widely deployed Least Congested Channel selection strategy (LCC).
\end{abstract}

Keywords-Channel Assignment; D2D Communications; Optimisation; Radio Resource Management; SDN; Wi-Fi Networks;

\section{INTRODUCTION}

With the massive proliferation of smart devices, wireless networks are facing the challenge of accommodating high data rates required by the increasing numbers of wireless users. As the wireless spectrum is limited, new techniques are needed to utilise this resource efficiently and optimise the capacity of the network. Recently, the concept of Device-to-Device (D2D) communications has emerged as an attractive solution to the spectrum efficiency problem in wireless networks [1-3]. In this concept, two nearby devices can communicate directly with each other without relying on network infrastructure entities such as the Wi-Fi access point (AP) or base station (BS) [4, 5]. In addition to Bluetooth and Wi-Fi Direct, D2D has been adopted in the 4G LTE-Advanced standard in 3GPP Release 12 under close proximity services (ProSe) [6,7]. Moreover, D2D is expected to be among the main concepts used in the Fifth Generation (5G) cellular networks [8].

This work has received funding from the European Union's Horizon 2020 Research and Innovation programme under Grant Agreement no. 644262 as part of the Wi-5 project.
By establishing direct communication between devices, D2D can relieve the rest of the wireless network from sharing the burden of supporting certain demanding applications (e.g., video sharing, gaming and proximity-aware social networking). This will improve the overall network performance in terms of throughput, energy efficiency, delay and fairness [5] while alleviating congestion and freeing spectrum for other users.

In Wi-Fi networks, where certain devices are in close proximity and can support applications without a direct connection to an AP, D2D can improve the spectral efficiency and decrease the load on the AP, especially when the data rate requirements are high (e.g., a mobile device that is streaming video contents to a smart TV). However, despite its promising features, D2D communications require coordinated management of radio resources in order to guarantee an acceptable link quality for each D2D connection. The management of radio resources for D2D communications is particularly important in dense Wi-Fi networks where wireless devices operate over unlicensed bands with a high probability of interference. Thus, the orchestration of establishing D2D connections should be the responsibility of the network operator rather than the communicated devices themselves.

The issues of Radio Resource Management (RRM) and interference for D2D communications in cellular networks have been the focus of current work in the literature [9-11], while little attention has been paid for those in Wi-Fi networks. Moreover, all related works assume that D2D connections are established following the self-organising approach. In [12], managing D2D communications in Wi-Fi networks has been partly addressed with a focus on enhancing Quality of Service (QoS) and energy efficiency. The Dynamically Synchronised Power Management (DSPM) scheme proposed in this work focuses on Wi-Fi Direct technology. DSPM tries to improve power efficiency and reliability by adjusting the sleep cycles and transmission slots according to the requirements of the different services using D2D communications. However, it does not address the problem of interference management in dense Wi-Fi networks.

In this paper, we utilise the Software-Defined Networking (SDN) $[13,14]$ concept to propose a centralised Wi-Fi management framework for D2D communications. SDN has emerged as an efficient and flexible network management concept where the control plane is decoupled from the data plane. This way, SDN can centralise the management operations 
for a domain into a single entity, often referred to as a Controller. Due to its flexibility, the SDN concept is currently adopted in wireless network management, including Wi-Fi networks. In our proposed framework, the controller monitors new data traffic to determine D2D flows. Once detected, our developed D2D connection algorithm is triggered to connect the two wireless devices. The D2D connection algorithm relies on a novel Channel Assignment Scheme to find an optimal channel configuration that allocates a channel for the D2D connection. At the same, it aims to minimise the effect of this new connection on the interference levels within a dense Wi-Fi network.

The rest of this paper is structured as follows. Section II describes the SDN-based Wi-Fi management framework and provides the details of the proposed algorithm for D2D and nonD2D traffic associations. Section III explains the AP channel assignment process implemented in our framework. Section IV presents the simulation setup and the scenarios designed to evaluate the performance of the proposed algorithm. Finally, Section V concludes the paper and discusses future work.

\section{SDN-BASED WI-FI MANAGEMENT FRAMEWORK}

In our work, we consider a dense Wi-Fi environment consisting of APs and wireless stations controlled by an SDN Controller, as illustrated in Fig. 1. The SDN-based framework proposed in this paper is based on the architecture presented in [15] and developed in the context of the H2020 EU Wi-5 (What to do With the Wi-Fi Wild West) project, which addresses spectrum congestion in Wi-Fi networks using SDN. This architecture, which will be available at the end of the project, is designed to provide efficient RRM solutions for a wide set of scenarios and use cases such as Airport/train station and Dense apartment building [16]. In this SDN-based framework, the management algorithms are implemented as applications on top of the Controller, which are triggered according to the network conditions and the characteristics of the flows exchanged within the Wi-Fi network.

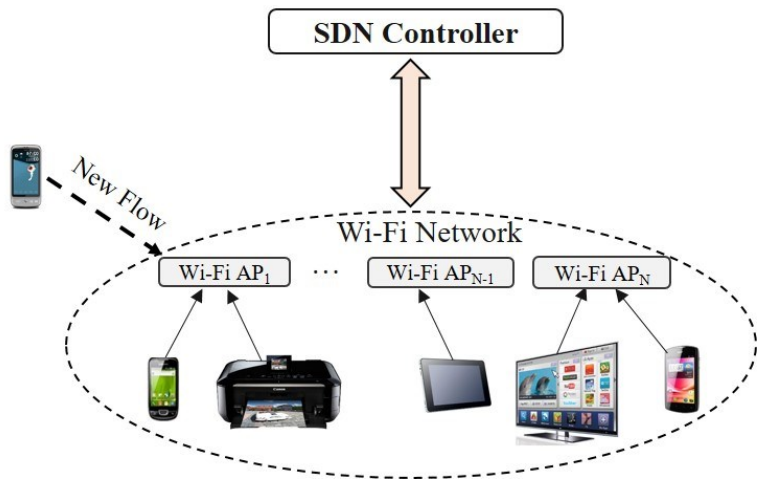

Fig. 1. Managing Wi-Fi Networks Using the SDN-Based Framework

The proposed SDN-Based framework relies on a Flow and Network Monitoring Module that resides inside the SDN Controller. This module takes regular measurements from the network and monitors the characteristics of new flows entering the network. The module uses the monitoring information it collects from the network and the data flows to trigger the appropriate management algorithm. Once the appropriate algorithm is triggered, a configuration is determined and applied to the Wi-Fi network, as illustrated in Fig. 2.

\section{A. Flow and Network Monitoring Module}

In Fig. 2, the Flow and Network Monitoring Module computes the following information: 1) the interference levels sensed through the APs; 2) the application type corresponding to the new flow; and 3) the current position of the source and the destination nodes. The detection of the application type can be performed by either proactively programming it into the SDN controller [17], or reactively inferring it through Machine Learning (ML) based strategies [18], which can be easily implemented in our proposed framework. Here, we assume that the information used by this functionality, to detect the application type, is available.

If the detected application corresponds to a D2D flow (e.g., a user needs to print a file on a Wi-Fi connected printer or stream video contents to a smart TV), the Controller triggers the establishment of a D2D connection. Examples of practical scenarios that involve D2D communications and can benefit from the proposed framework are: 1) a home networking scenario in which the station pairs correspond to a smart TV and a user; and 2) a home or an office networking scenario where the station pairs are, respectively, a wireless printer and a user. Many other examples can be envisioned.

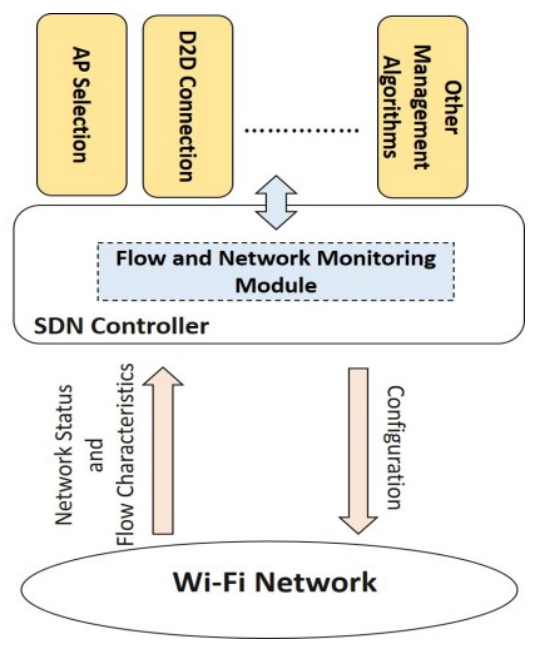

Fig. 2. SDN-Based Wi-Fi Management Framework for Triggering Appropriate Algorithm for D2D and non D2D traffic

On the other hand, if the detected application does not need a D2D configuration (e.g., a user watches a video on YouTube), the Controller triggers an AP connection. In this case, the source and the destination are, respectively, the selected AP and the user who is watching the video. Moreover, the Flow and Network Monitoring Module provides the interference levels sensed around the AP. The approach implemented in the AP selection process is based on the Received Signal Strength Indicator (RSSI) as recommended by the IEEE 802.11 standard. Finally, the AP/user association process assigns the selected AP to the new flow and runs the detected application.

\section{B. D2D Connection Algorithm}

The D2D Connection Algorithm dynamically establishes a connection between devices in close proximity once the Flow 
and Network Monitoring Module detects a traffic flow that follows a D2D traffic pattern.

First, the algorithm elects one of the D2D nodes as a Wi-Fi AP, which we refer to as a soft-AP. Once a soft-AP is elected, the algorithm triggers a Channel Assignment Process that calculates the best channel configuration to establish the D2D communication between the two devices while minimising the effect of this connection on the overall performance of the network. Since the D2D algorithm will operate in dense Wi-Fi environments, it is important to find a channel assignment configuration that has minimal impact on the overall interference levels within the Wi-Fi network. Fig. 3 provides an overall description of the proposed D2D Connection algorithm.

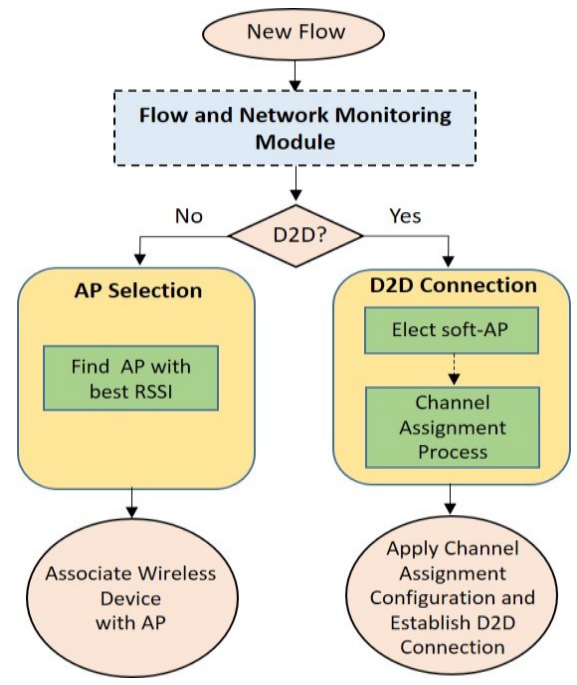

Fig. 3. D2D Connection Algorithm

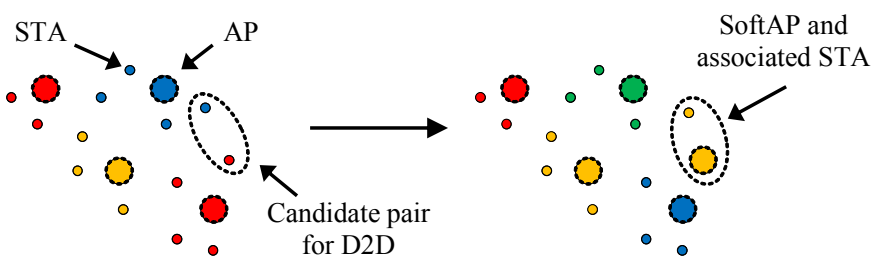

Fig. 4. Optimal channel assignment configuration including a soft-AP

Fig. 4 describes an example where the Channel Assignment Process is triggered to find an optimal channel configuration when a soft-AP is elected by the D2D Connection algorithm. The left side of the figure represents the optimal channel assignment configuration across the Wi-Fi network that consists of 4 APs (the big circles) using 3 RF channels denoted by different colours (red, blue and yellow), and different STAs (the small circles), before D2D traffic is detected. Once a D2D traffic is detected, the D2D Connection algorithm is triggered, which in its turn launches the Channel Assignment Process, which is explained later in Section III, to find a new optimal channel assignment configuration for the Wi-Fi network that consists of 5 APs (4 fixed APs and 1 soft-AP) after establishing the D2D connection. The new channel configuration is illustrated on the right side of the figure where new channels are assigned to the APs. Note that this also force their connected devices to change channels in order to continue their services.
Furthermore, it is worth noting from Fig. 4, that the new channel assignment process only affects the assigned channels of APs and STAs in close range. Once an optimal configuration is found, the soft-AP is assigned an RF channel, and a connection is established between the two nearby devices. Note that for accurate performance for the channel assignment process, the D2D Connection algorithm needs to use monitoring data collected by the Flow and Network Monitoring Module in order to measure the interference levels around the soft-AP.

\section{The ChAnNEl AssignMENT Process}

In this section, we provide a comprehensive description of the Channel Assignment Process executed in the D2D Connection algorithm. We consider $N$ Wi-Fi APs, based on the IEEE 802.11 standard, that operate on $F$ RF channels including $F_{n o n}$ of them not-overlapping each other. We also assume $N>$ $F_{\text {non }}$ (i.e., there is channel overlapping and interference problem in the network). For instance, $F=11$ and $F_{\text {non }}=3$ in the North American regulated IEEE $802.112 .4 \mathrm{GHz}$ band where $N \geq 4$ is the starting point of channel overlapping and densification problem. The Channel Assignment Process relies on the following information: 1) the topology of the network and the arrangement of the APs; 2) the current channel assignments across all APs; and 3) the characteristics of the IEEE 802.11 RF channels and their impact on the interference due to a combination of orthogonality and overlapping of the channels.

We define the network topology matrix $G \in\{0,1\}^{N \times N}$, where:

$$
g_{i j}=\left\{\begin{array}{c}
1, \quad \begin{array}{r}
\text { average power strength of } A P_{i} \text { around } A P_{j} \\
\text { exceeds a given threshold }
\end{array} \\
0, \quad \text { otherwise }
\end{array}\right.
$$

We define the channel assignment matrix $A \in\{0,1\}^{F \times N}$, where:

$$
a_{i j}=\left\{\begin{array}{lc}
1, & \text { if channel } i \text { is assigned to } A P_{j} \\
0, & \text { otherwise }
\end{array}\right.
$$

We also define $I \in \mathbb{R}^{N \times F}$, the matrix of the interference for $N$ APs and $F$ available channels, where $I_{i, j}$ is the accumulation of the interference levels detected at other APs as a result of assigning channel $j$ to AP $i$. I embodies the interference impact of assigning each RF channel to each AP given the channel assignment of other APs and the overlap between them. This relies on the history of the measurements collected from the APs and processed at the central controller (i.e., I as a-priori information). Finally, we define $U$ as an objective function that represents the interference levels detected by all APs due to their current channel assignment configuration formulated as follows:

$$
W=G \times A^{T}, \quad U=W . I
$$

Where ' $\times$ ' represents the matrix multiplication, $A^{T}$ is the transpose matrix of $A$, and ' ' denotes element-wise multiplication of the matrices. $U$ is actually obtained by taking into account the arrangement of the APs, reflected in $G$, and the channel assignment represented by $A$ alongside the actual impact of the channel selection at each AP from the interference point of view represented by $I$. Since $I$ is a matrix with real values $\left(I \in \mathbb{R}^{N \times \mathrm{F}}\right), U$ in (3) will also be a real matrix $\left(U \in \mathbb{R}^{N \times \mathrm{F}}\right)$. We 
can describe $U$ as an objective function, which represents the magnitude of the interference in the whole system and encompasses the scale by which APs are conflicting with each other in each channel represented by $G \times A^{T}$, as follows:

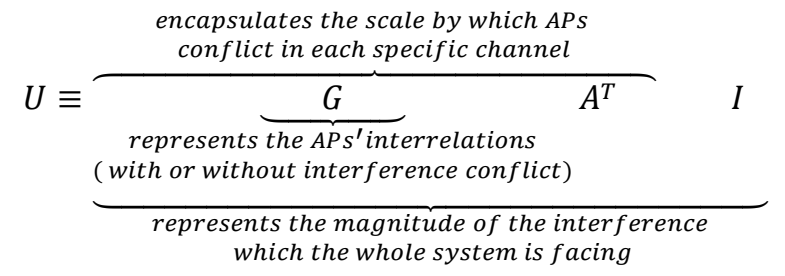

To clarify this concept, a simple network with $N=5$ APs and $F=3 \mathrm{RF}$ channels (coloured with red, blue and yellow) is shown in Fig. 5. We assume that AP1 is not in the contention range of AP4 and AP5, hence the corresponding elements in $G$ will be zero. Matrix $A$ represents the allocated channels to the APs and $W=G \times A^{T}$ embodies the scale of the conflict from each AP with regards to each channel. For example, given the channel assignment and the arrangement of AP1, AP2, AP3 and AP5, $w_{41}=0$ indicates no conflict for the channel coloured with red if it is assigned to AP4, while the value $w_{43}=2$ indicates a higher scale of conflict if the channel coloured with yellow is assigned to AP4. By applying the actual interference of the channels considering their overlap and orthogonality through $I$ in (3), the objective function $U$ provides the network-wide interference quantities, which need to be minimised through an optimised channel assignment.

We define $A^{*}$ as the optimised channel assignment matrix that provides the minimum accumulated interference levels and can be obtained as follows:

$$
A^{*}=\min _{A} \sum_{i \leq N} \sum_{j \leq \mathrm{F}} u_{i, j}
$$

The power and interference values (i.e., the elements of $U$ in (5)) are considered to be real values. The channel assignment optimisation problem and constraints can be expressed by:

$$
\left\{\begin{array}{c}
A^{*}=\min _{A} \sum_{\text {all }}\left(G \times A^{T} . I\right) \\
A \in\{0,1\}^{F \times N} \\
|| A(:, i) \|_{1}=1 \\
\forall a_{i j} \in A: \sum \sum a_{i j}=N
\end{array}\right.
$$

Where $\|\cdot\|_{1}$ represents 1 -norm, the summation of the elements in each column of $A$. The constraints in (6) are based on the fact that each AP will be assigned only one channel and in total exactly $N$ channel selections should be made for $N$ APs.

$A=\left(\begin{array}{ccc}a_{11} & \cdots & a_{1 N} \\ \vdots & \ddots & \vdots \\ a_{F 1} & \cdots & a_{F N}\end{array}\right) \rightarrow x=\left(\begin{array}{c}x_{1} \\ \vdots \\ x_{N} \\ \vdots \\ x_{(F-1) \times N+1} \\ \vdots \\ x_{F \times N}\end{array}\right) \equiv\left(\begin{array}{c}a_{11} \\ \vdots \\ a_{1 N} \\ \vdots \\ a_{F 1} \\ \vdots \\ a_{F N}\end{array}\right)$

By element-wise multiplication of matrices in (6) and representing all elements of matrix $A$ in the form of a vector of unknown values, $x \in\{0,1\}^{N * F \times 1}$, as shown in equation (7), the optimisation problem presented in (6) can be solved using a binary Integer Linear Programing (ILP) as follows:

$$
\begin{aligned}
& x^{*}=\min _{x} c^{T} x \\
& B x=b \\
& x_{i} \in[0,1], \forall i: 1 \leq i \leq N \times F \\
& c=f(G, I), \quad b=1^{N \times 1},
\end{aligned}
$$

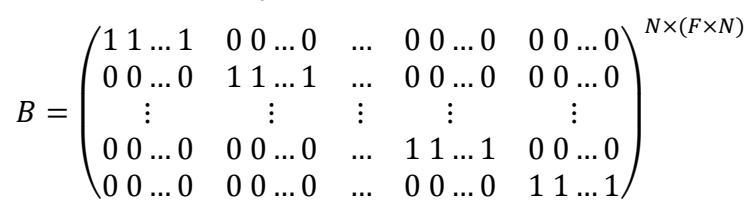

Where $c$ is a coefficient matrix resulting from the elementwise expansion of (6), and $x$ provides the desired channel assignment (i.e., the elements of matrix $A^{*}$ ). The channel assignment matrix $A$, in (6), has just one non-zero value in each row corresponding to the assigned channel. Matrix $B$ reflects the constraints defined for $A$ in (6) over the values of $x$ in (8).

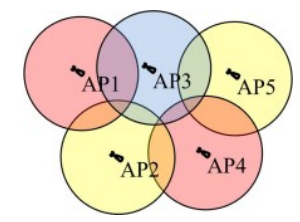

$$
G=\left[\begin{array}{lllll}
0 & 1 & 1 & 0 & 0 \\
1 & 0 & 1 & 1 & 1 \\
1 & 1 & 0 & 1 & 1 \\
0 & 1 & 1 & 0 & 1 \\
0 & 1 & 1 & 1 & 0
\end{array}\right], A^{T}=\overbrace{\left(\begin{array}{lll}
1 & 0 & 0 \\
0 & 0 & 1 \\
0 & 1 & 0 \\
1 & 0 & 0 \\
0 & 0 & 1
\end{array}\right)}^{B} \quad Y W=\left(\begin{array}{lll}
0 & 1 & 1 \\
2 & 1 & 1 \\
2 & 0 & 2 \\
0 & 1 & 2 \\
1 & 1 & 1
\end{array}\right)
$$

Fig. 5. An example describing the application of $G, A$ and $W$

\section{Evaluation Scenario And Simulation Results}

\section{A. Simulation Setup and Evaluation Strategy}

In this section, we evaluate the performance of our SDNbased framework in a dense Wi-Fi environment that includes D2D communications alongside native $\mathrm{Wi}-\mathrm{Fi}$ infrastructure. The simulations are run using MATLAB. We investigate the performance of the network with D2D capability and compare it to the infrastructure mode of the same network (i.e., when the SDN controller always triggers the AP selection process). The simulation settings and parameters are summarised in Table I.

TABLE I - SUMMARY OF THE SIMULATION PARAMETERS

\begin{tabular}{|c|c|}
\hline Simulation Area & $250 \mathrm{~m} \times 250 \mathrm{~m}$ \\
\hline Number of Wi-Fi APs & 4 \\
\hline Minimum distance between APs & $50 \mathrm{~m}$ \\
\hline AP Transmit Power & Assigned between $10 \mathrm{dBm}$ to $25 \mathrm{dBm}$ \\
\hline Users' stations deployment & $\begin{array}{c}\text { Randomly at a minimum distance of } \\
1 \mathrm{~m} \text { from each other and from the APs }\end{array}$ \\
\hline
\end{tabular}

It is worth noting that the simulated network will be further densified by the D2D connections. Once assigned, the transmit power of the AP remains unchanged during the simulation (i.e., there is no transmit power control). Finally, we adopted a large 


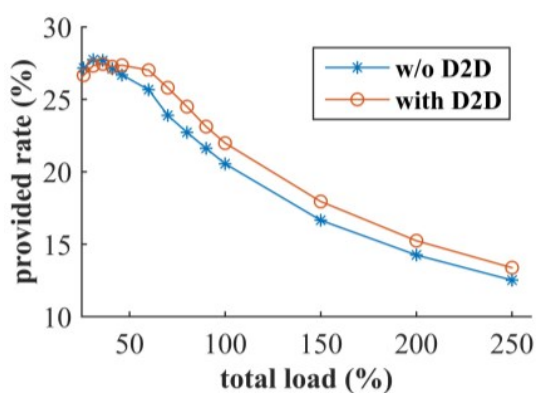

Fig. 6. Provided Rate with and without D2D Capability

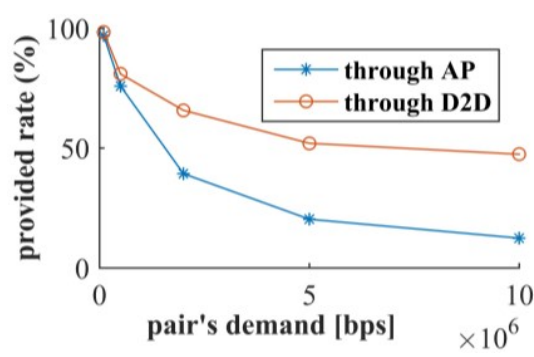

Fig. 7. Provided Rate for D2D pairs with and without D2D Capability

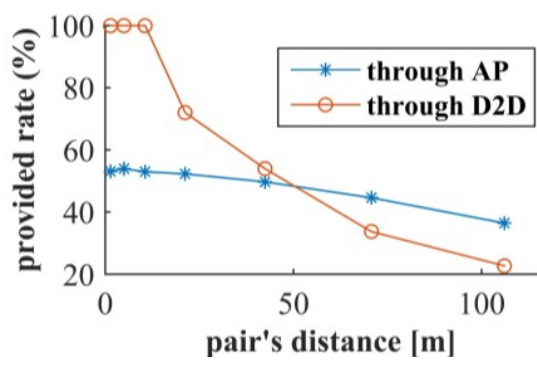

Fig. 8. Provided Rate in function of Pair's Distance scale path loss model with the exponent set to 2.5 , a fixed noise level at $-95 \mathrm{dBm}$ and the threshold in (1) set to $-78 \mathrm{dBm}$.

When D2D is deployed (i.e., a new D2D connection is established), one device of each pair is transformed into a softAP and the other one will be associated with it. During D2D deployments, the channel assignment process is reconfigured in order to allocate new channels to the APs and soft-APs. On the other hand, in the case of the infrastructure mode, the channel assignment process is executed only at the beginning of the simulation to configure the 4 fixed APs forming the native WiFi infrastructure mode.

The provided rate illustrated in our performance analysis is defined as the served rate divided by the required rate demonstrated. The required rate varies from $100 \mathrm{kbps}$ to $10 \mathrm{Mbps}$ and depends on application type. Furthermore, in our evaluation, we compare the performance of our SDN-based channel assignment strategy against the common Least Congested Channel (LCC) selection mechanism which is widely adopted for this purpose [19]. In LCC, each AP acquires a suitable channel based on the neighbouring APs' channels.

\section{B. Simulation Results Analysis}

In the following, we firstly investigate the performance of the Wi-Fi network with D2D capability using our SDN-based framework in comparison to the infrastructure mode without D2D capability. Secondly, we study the performance of our Channel Assignment Process in the context of D2D communications.

For the first evaluation objective, we compare the average provided rates for users in the presence of various loads in the network with and without D2D capability, which is provided by our framework. We assume a scenario where the number of users varies between 60 and 200 to create a complete range of load compared to the capacity of the exemplified network. In order to provide a consistent comparison, we assume that there are 5 established D2D pairs in all instances of the loads. Fig. 6 shows that the infrastructure mode is capable of providing the maximum efficiency for the users in an uncongested network. However, when network becomes more congested, D2D capability provides better performance.

Fig. 7 shows the rates that the network could provide to potential D2D pairs via the infrastructure mode and when the D2D capability is available. It can be seen that the Wi-Fi infrastructure mode can satisfy the users with low data rates (more than $75 \%$ satisfaction for users with up to $500 \mathrm{Kbps}$ required data rate). However, as the required data rate increases, the network performance starts to drop (just around $10 \%$ satisfaction for users with $10 M b p s$ required data rate). On the other hand, with a D2D capability, the network can provide better performance for D2D pairs, especially when the data rate requirements increase (up to $55 \%$ satisfaction for a pair demanding 10Mbps data rate).

Fig. 6 and 7 demonstrate that the D2D capability provided by our proposed framework helps to increase the network capacity and improve its performance especially in a spectrum congested environment. These results also show that data requirements demands, especially when high, are better served when the network offers a D2D capability. However, as shown in Fig. 8, the performance of the network in this context also depends heavily on the distance between the D2D pairs.

Fig. 8 shows that paired users who are far from each other are better-off using the infrastructure mode (i.e., go through the AP). Thus, there is a trade-off between the impact of the path loss in a D2D connection and the restriction of the capacity of the APs in the infrastructure mode.

For the second evaluation objective, we compare the performance of the network in terms of the signal-tointerference-plus-noise ratio (SINR) in the presence of D2D communications. As discussed previously, each D2D soft-AP is assigned a dedicated channel, which adds to the densification of the network and might increase the overall interference, hence affecting its performance. The increased network interference results in a degradation of the average SINR in the network for all users. Fig. 9 shows the impact of the interference in the form of average SINR in the case of LCC and our proposed Channel Assignment Process. It can be seen from Fig. 9 that our SDNbased approach achieves better performance than LCC's.

Finally, Fig. 10 illustrates the improvement of the achieved data rate for all users in the network with the two channel assignment approaches (LCC and SDN-based). Fig. 10 clearly shows that our SDN-based framework outperforms LCC by more than $6 \%$ allowing the network to accommodate more D2D connections. It can be seen that when the LCC approach is used, the network performance reaches its maximum when serving 20 D2D connections. As the number of D2D connections increases, the network performance degrades and the network becomes unable to cope where the number of $\mathrm{D} 2 \mathrm{D}$ connections exceeds 40. On the other hand, for the same number of D2D users, our SDN-based framework allows the network to provide a higher data rate. This proves that our SDN-based framework 
manages the interference created by D2D connections much better through the Channel Assignment Process, which

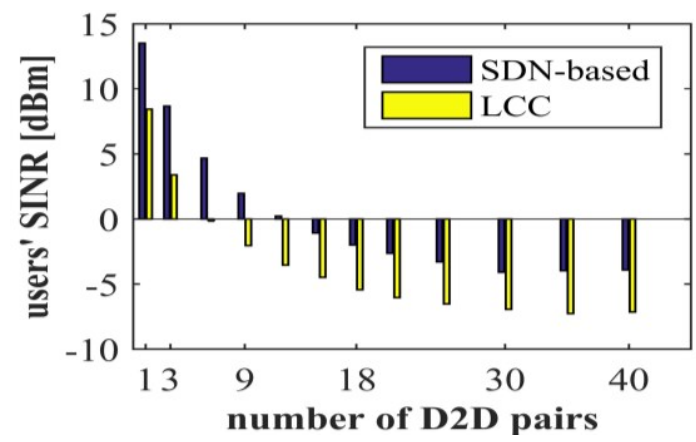

Fig. 9. Reception Quality in terms of SINR - Impact of the D2D Deployment Size

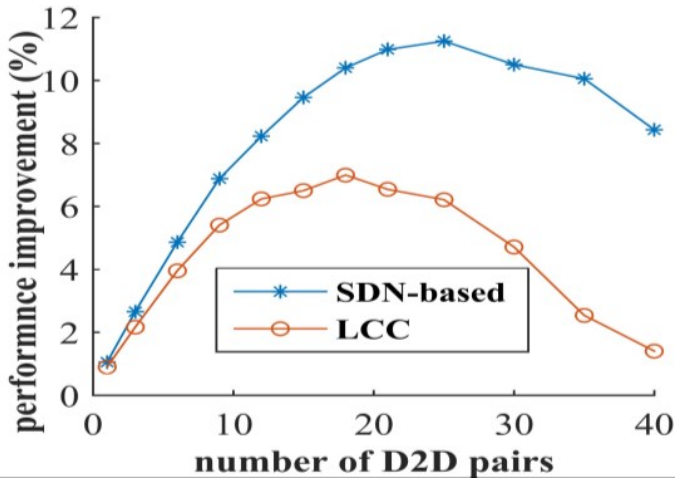

Fig. 10. D2D Performance - Impact of the Interference Management Approach

provides optimal channel assignment configuration.

\section{CONCLUSIONS AND FUTURE WORKS}

In this paper, we proposed a centralised management framework for D2D communications in dense Wi-Fi networks using SDN-based centralised controller in synergy with a novel AP channel assignment process. The proposed framework can detect D2D traffic flows and establish direct connections between D2D devices. Simultaneously, it reduces the effect of these communications in terms of the overall capacity of the network and the interference on other Wi-Fi users. Simulation results showed that our proposed framework highly improves the network performance when D2D connections are deployed. It has also been demonstrated how the flexibility and the centralised nature of the SDN controller can provide all the information required for a better radio resources management, which minimises the effect of the interference caused by the deployed D2D connections.

For future work, we will focus on improving the Flow and Network Monitoring Module. The improvements include detecting D2D communications that involve mobile devices and the possibility of re-establishing the infrastructure mode connection when the involved devices are not in close proximity. We will also consider improvements to the AP selection algorithm for non-D2D traffic by replacing the RSSI selection metric with QoS-related metrics, which reflect the suitability of an AP to a specific user according to the available resources of the AP and the user's required quality.

\section{REFERENCES}

[1] "H2020 EU Project 671704 CHARISMA (Converged Heterogeneous Advanced 5G Cloud-RAN Architecture for Intelligent and Secure Media Access)," 2015. [Online]. Available: http://www.charisma5g.eu/. [Accessed 22 Mar 2016].

[2] "H2020 EU Project 644526 iCIRRUS (intelligent Converged network consolIdating Radio and optical access aRound USer equipment)," 2015. [Online]. Available: http://www.icirrus-5gnet.eu/. [Accessed 22 Mar 2016].

[3] D. Camps-Mur, A. Garcia-Saavedra and P. Serrano, "Device-to-Device Communications with WiFi Direct: Overview and Experimentation," IEEE Wireless Communications, vol. 20, no. 3, pp. 96-104, 2013.

[4] M.N. Tehrani, M. Uysal and H. Yanikomeroglu, "Device-to-Device Communication in 5G Cellular Networks: Challenges, Solutions, and Future Directions," IEEE Communication Magazine, vol. 52, no. 5, pp. 86-92, 2014.

[5] A. Asadi, Q. Wang and V. Mancuso, "A Survey on Device-to-Device Communication in Cellular Networks," IEEE Communications Surveys \& Tutorials, vol. 16, no. 4, pp. 1801-1819, 2014.

[6] X. Shen, "Device-to-Device Communication in 5G Cellular Networks," IEEE Network, vol. 29, no. 2, pp. 2-3, 2015.

[7] 3GPPP, "3GPP Specification: 22.803 Feasibility study for Proximity Services (ProSe)" 17 Sept 2014. [Online]. Available: http://www.3gpp.org/DynaReport/22803.htm. [Accessed 15 July 2015].

[8] M.H. Eiza, Q. Ni and Q. Shi, "Secure and Privacy-Aware Cloud-Assisted Video Reporting Service in 5G Enabled Vehicular Networks," IEEE Trans. Vehicular Technology, DOI: 10.1109/TVT.2016.2541862, Mar 2016.

[9] L. Lei, Z. Zhong, C. Lin, X. Shen, "Operator Controlled Device-to-Device Communications in LTE-Advanced Networks," IEEE Wireless Communications, vol. 19, no. 3, pp. 96-104, 2012.

[10] S. Shalmashi, G. Miao and S.B. Slimane, "Interference Management for Multiple Device-to-Device Communications Underlaying Cellular Networks," in Proc. PIMRC, pp. 223-227, London 2013.

[11] L. Militano, M. Condoluci, G. Araniti, A. Molinaro, A. Iera, G. M. Muntean, "Single Frequency-Based Device-to-Device-Enhanced Video Delivery for Evolved Multimedia Broadcast and Multicast Services," IEEE Transactions on Broadcasting, vol.61, no.2, pp.263-278, 2015.

[12] K-W. Lim, W-S. Jung and Y-B. Ko, "Energy Efficient Quality-of-Service for WLAN-based D2D Communications," Ad hoc Networks, vol. 25, pp. 102-116, 2015.

[13] N. McKeown, T. Anderson, H. Balakrishnan, G. Parulkar, L. Peterson, J. Rexford, S. Shenker and J. Turner, "OpenFlow: Enabling Innovation in Campus Networks," ACM SIGCOMM Computer Communication Review, vol. 38, no, 2, pp. 69-74, 2008.

[14] C. Monsanto, J. Reich, N. Foster, J. Rexford and D. Walker, "Composing Software-Defined Networks," in Proc. USENIX, Lombard, US, 2013.

[15] F. Bouhafs (Editor), "Wi-5 initial architecture", Deliverable D2.4 of Wi5 project, Dec. 2015, available at http://www.wi5.eu/.

[16] I. Berberana (Editor), "Wi-5 use cases and requirements", Deliverable D2.3 of Wi-5 project, Dec. 2015, available at http://www.wi5.eu/.

[17] R.Wallner and R.Cannistra, "An SDN Approach: Quality of Service using Big Switch's Floodlight Open-source Controller," Proc. The Asia-Pacific Advanced Network, vol. 35, p. 14-19, 2013.

[18] T.T.T. Nguyen, G. Armitage, P. Branch and S. Zander, "Timely and Continuous Machine-Learning-based Classification for Interactive IP Traffic," IEEE/ACM Transactions on Networking, vol. 20, no. 6, pp. 1880-1894, 2012.

[19] M. Achanta, "Method and Apparatus for Least Congested Channel Scan for Wireless Access Points," US Patent No. 20060072602, Apr. 2006. 